TIT/HEP-390

April '98

\title{
On the cosmological domain wall problem in supersymmetric models
}

\author{
Tomohiro Matsuda 円 \\ Department of physics, Tokyo institute of technology \\ Oh-okayama, Meguro, Tokyo 152-8551, Japan
}

\begin{abstract}
Many models for dynamical supersymmetry breaking have the domain wall solution of the Kovner-Shifman type and therefore contain the potential cosmological domain wall problem. We show that the degeneracy of the vacua is lifted in any model in which the cosmological constant is fine-tuned to zero through an explicit $Z_{n}^{R}$ symmetry breaking constant in the superpotential. In this respect, we do not have to add unnatural non-renormalizable terms which explicitly break the symmetry. We also discuss the conditions for the safe decay of the walls for several concrete examples.
\end{abstract}

${ }^{1}$ matsuda@th.phys.titech.ac.jp 


\section{Introduction}

In general many models for supersymmetry breaking have domain wall solutions of the Kovner-Shifman type[1] in their dynamical sectors where the discrete symmetry is implemented by their anomalies. A domain wall configuration may also appear as the

consequence of spontaneous symmetry breaking of the explicit $Z_{n}^{R}$ symmetry which is imposed by hand in order to solve the phenomenological difficulties such as the $\mu$-problem [2] or the cosmological moduli problem[3]. These domain walls are generally dangerous from the cosmological point of view. If the Universe undergoes a phase transition associated with the spontaneous symmetry breaking, domain walls will inevitably form and in most cases these domain walls are very dangerous for the standard evolution of the Universe.

In this paper we study the properties of the Kovner-Shifman domain walls in general supersymmetric models. We show that the degeneracy of the vacua is always lifted by a constant term when supergravity is turned on. The magnitude of the energy difference is estimated to be $\sim \sigma^{2} / M_{p}^{2}$ where $\sigma$ is the surface energy density of the wall. This value is sufficient for the safe decay of the cosmological domain wall provided $\sigma$ is larger than $\left(10^{5} \mathrm{GeV}\right)^{3}$. Although the model is technically non-generic because it includes a single term which explicitly breaks the $Z_{n}^{R}$ symmetry, it is still a reasonable model for a vanishing cosmological constant.

The basic idea is very similar to the well-known mechanism for the mass generation of the R-axion [4]

\section{Kovner-Shifman Domain walls}

In this section we explain the basic idea of the Kovner-Shifman domain wall[1]. The Kovner-Shifman domain wall is a configuration which preserves a half of the supersymmetry. The key ingredient is the central extension of the $\mathrm{N}=1$ superalgebra

$$
\left\{\bar{Q}_{\dot{\alpha}}, \bar{Q}_{\dot{\beta}}\right\}=-2 i\left(\sigma^{0}\right)_{\dot{\alpha}}^{\gamma} \int d^{3} x\left\{\bar{D}_{\dot{\beta}} \bar{D}^{\dot{\delta}} J_{\gamma \dot{\delta}}\right\}_{\theta=0}
$$

The contribution to the central extension is due to the non-conservation of the current multiplet $J$. The lowest component of $J$ is the $U(1)_{R}$-current which is broken classically 
(in Wess-Zumino model with more than two terms in the superpotential) or by quantum anomaly $\left(U(1)_{R}\right.$ anomaly in gauge theories). Although the current multiplet $J$ have anomaly, the supersymmetry current is conserved thus supersymmetry is still unbroken. What is not conserved in this case is not the supersymmetry current but the superconformal current.

Domain wall in non-gauge theories

The simplest example for the Kovner-Shifman domain wall is the Wess-Zumino model with only a superfield $\Phi$. In non-gauge theories, the presence of the central extension can be seen at the tree level. The Wess-Zumino Lagrangian in terms of a superfield $\Phi$ has the following form,

$$
\begin{aligned}
L & =\frac{1}{4} \int d^{4} \theta \Phi \Phi+\left[\frac{1}{2} \int d^{2} \theta W(\Phi)+h . c .\right] \\
W(\Phi) & =\mu^{2} \Phi-\frac{\lambda}{3} \Phi^{3} .
\end{aligned}
$$

When $\mu=0$, the Lagrangian is invariant under the $U(1)_{R}$ rotation

$$
\begin{aligned}
d \theta & \rightarrow e^{-i \alpha} d \theta \\
\Phi & \rightarrow e^{\frac{2 i \alpha}{3}} \Phi .
\end{aligned}
$$

However, if $\mu \neq 0$, this $U(1)_{R}$ invariance is broken and only the discrete part $Z_{2}^{R}$ persists,

$$
\begin{aligned}
d^{2} \theta & \rightarrow-d^{2} \theta \\
\Phi & \rightarrow-\Phi
\end{aligned}
$$

When $<\Phi>\neq 0$, the $Z_{2}^{R}$ symmetry is spontaneously broken and the corresponding domain wall which interpolates between two degenerate vacua

$$
\begin{aligned}
<\Phi> & = \pm \mu / \sqrt{\lambda} \\
<W> & =\mp \frac{2}{3} \frac{\mu^{3}}{\sqrt{\lambda}}
\end{aligned}
$$

appears. In this case the supersymmetric current multiplet $J$ has the anomaly of the following form,

$$
\bar{D}^{\dot{\alpha}} J_{\alpha \dot{\alpha}}=\frac{1}{3} D_{\alpha}\left[3 W-\Phi \frac{\partial W}{\partial \Phi}\right] .
$$


Substituting the anomaly equation into the central extension of the superalgebra(2.1), one obtains

$$
\left\{Q_{\alpha}, Q_{\beta}\right\}=4(\vec{\sigma})_{\alpha \beta} \int d^{3} x \vec{\nabla}\left[\bar{W}-\frac{1}{3} \bar{\Phi} \frac{\partial \bar{W}}{\partial \bar{\Phi}}\right]_{\bar{\theta}=0}
$$

where the right hand side of the equation is related to the central charge of the wall configuration therefore it represents the surface energy density of the wall configuration. It is apparent that the contribution is non-zero when the domain wall interpolates the two different vacuum configurations of eq.(2.5).

The domain wall solution for such non-gauge theories can easily be extended to more general theories such as an effective theory of SQCD or some other complicated theories. For later convenience we show one of such examples which contains explicit cut-off parameter of the theory and have the superpotential with discrete $Z_{n}^{R}$ symmetry,

$$
\begin{aligned}
d^{2} \theta & \rightarrow d^{2} \theta e^{-\frac{2 a \pi i}{n}} \\
X & \rightarrow X e^{\frac{2 \pi i}{n}} .
\end{aligned}
$$

The superpotential takes a form

$$
W=\sum_{k=0}^{\infty} \frac{\lambda_{k}}{n k+a} X^{n k+a} M^{-n k-a+3},
$$

where $\lambda_{k}$ and $M$ denotes the coupling constants and the cut-off scale of the model. $a$ is an integer which will be fixed in the corresponding phenomenological models. If the coupling constants are assigned so that the scalar potential exhibits non-trivial minima at $<X>\sim M e^{\frac{2 \pi i}{n} k}(k=1, . . n)$, the discrete symmetry is spontaneously broken. A domain wall solution which interpolates the degenerated $Z_{n}^{R}$ vacuum configurations appears and the wall have the surface energy density of order $M^{3}$.

Domain wall in gauge theories

Now let us discuss the supersymmetric QCD(SQCD) with or without the superpotential. As we have stated above, this theory have an anomaly which contributes to the central extension. The Lagrangian is

$$
L=\left[\frac{1}{4 g^{2}} \int d^{2} \theta \operatorname{Tr} \mathcal{W}^{2}+\text { h.c. }\right]+\frac{1}{4} \int d^{2} \theta\left[\bar{Q}^{i} e^{V} Q^{i}+\overline{\widetilde{Q}}^{i} e^{V} \tilde{Q}^{i}\right] .
$$


In superfield notation the anomaly in the supermultiplet of current is

$$
\begin{aligned}
\bar{D}^{\dot{\alpha}} J_{\alpha \dot{\alpha}}= & \frac{1}{3} D_{\alpha}\left\{\left[3 W-\sum_{i} Q_{i} \frac{\partial W}{\partial Q_{i}}\right]\right. \\
& {\left.\left[\frac{3 T(G)-\sum_{i} T\left(R_{i}\right)}{16 \pi^{2}} \operatorname{Tr}^{2}+\frac{1}{8} \sum_{i} \gamma_{i} Z_{i} \overline{(D)^{2}}\left(\bar{Q}_{i} e^{V} Q_{i}\right)\right]\right\} }
\end{aligned}
$$

where in SQCD, $T(G)=N_{c}$ and $T\left(R_{i}\right)=1$ for each flavor. Substituting the anomaly equation into the superalgebra(2.1) and using the Konishi anomaly equation, one gets

$$
\left\{\bar{Q}_{\alpha}, \bar{Q}_{\beta}\right\}=4(\vec{\sigma})_{\dot{\alpha} \dot{\beta}} \int d^{3} x \vec{\nabla}\left[W-\frac{T(G)-\sum_{i} T\left(R_{i}\right)}{16 \pi^{2}} \operatorname{Tr}^{2}\right]_{\theta=0} .
$$

For pure SYM without matter field, it is easy to find that the domain wall have nonzero central charge. The contribution to the central charge appears because gaugino condensation has the phase. To be more precise, the surface energy density takes a

form $\Lambda^{3}\left|1-e^{\frac{2 \pi i}{N_{c}}}\right|$ for nonchiral-nonchiral configurations or $\Lambda^{3}\left|0-e^{\frac{2 \pi i}{N_{c}}}\right|$ for chiral-nonchiral configuration. For $N_{f}<N_{c}$ SQCD the domain wall solution is formulated for the effective superpotential which looks like a extended Wess-Zumino model. It is easy to understand from eq. (2.12) that there is always a contribution to the central charge as far as gaugino condensation is non-zero. (Note that the coefficient in front of gaugino condensation does not vanish for $N_{f}<N_{c}$.) Although the contribution from anomaly vanishes for $N_{f}=N_{c}$ massless SQCD, the central charge does not vanish for massive SQCD because there is a contribution from the mass term [5].

\section{Cosmological problem of the Kovner-Shifman do- main wall}

In this section we discuss the cosmological problem of Kovner-Shifman domain walls and find their solutions.

It is well known that whenever the Universe undergoes a phase transition associated with the spontaneous symmetry breaking, domain walls will inevitably form. In most cases the domain walls are dangerous for the standard evolution of the universe.

As we have discussed in the previous section, the models for dynamical supersymmetry breaking or the models with explicit $Z_{n}^{R}$ symmetry should have the Kovner-Shifman domain wall solution. 


\section{Hidden sector models}

As the first example, let us consider the hidden sector models in which the supersymmetry breaking is triggered by gaugino condensation in the hidden sector. The typical scale of the surface energy density of the domain wall is related to gaugino condensation in the hidden sector and is estimated to be $\sim 10^{12} \mathrm{GeV}$ which is high enough to avoid the production of the cosmological domain wall during the reheating of the Universe.

\section{Gauge mediated supersymmetry breaking models}

The second example is the gauge mediated supersymmetry breaking model[7]. Although this type of models have so many variants, most of the models are inspired by the dynamical analysis of SQCD with $N_{f} \leq N_{c}$ fundamental fields. In this respect, here we may focus our attention only to SQCD with $N_{f} \leq N_{c}$ without loss of generality. Contrary to the hidden sector models, the surface energy density of the domain wall in the dynamical sector is very low. The walls can easily be produced during the reheating of the Universe thus needs some mechanism which makes the wall collapse in the proper time scale. The pressure which is required for the safe decay of the wall must be induced by the explicit breaking of the degeneracy of the vacuum energy. In the crudest estimate, the required value for the pressure is [6]

$$
\epsilon \geq \frac{\sigma^{2}}{M_{p}^{2}}
$$

where $\epsilon$ represents the pressure corresponding to the difference of the energy density between the corresponding vacua. $\sigma$ represents the surface energy density of the domain wall.

The most natural and simplest candidate for the source of $\epsilon$ is the constant term which arises from the general requirement that the cosmological constant must be canceled in any successful theory of dynamical supersymmetry breaking. In supergravity theories, at the tree level the scalar potential takes the following form

$$
\begin{aligned}
V & =V_{D}+V_{F} \\
V_{D} & =\frac{1}{2} g^{2} D^{a} D^{a} \\
V_{F} & =e^{\frac{K}{M_{p}^{2}}}\left(\left[W_{i}+\frac{K_{i}}{M_{p}^{2}} W\right] K_{i j^{*}}^{-1}\left[W_{j^{*}}^{*}+\frac{K_{j^{*}}}{M_{p}^{2}} W^{*}\right]-3 \frac{\left|W^{2}\right|}{M_{p}^{2}}\right),
\end{aligned}
$$

where $W$ and $K$ are the superpotential and the Kähler potential. In general models 
for supersymmetry breaking, a constant term $W_{0}$ is added and is adjusted to cancel the vacuum energy. Because we are assuming that $W_{0}$ is a constant, this fine tuning works only for one vacuum. I When the cancelation occurs for the $k_{0}$-th vacuum, the situation is schematically given as:

$$
\begin{aligned}
V_{\text {True }} & =|F|^{2}-3 \frac{\left|W_{0}+\Lambda^{3} e^{\frac{2 \pi i}{n} k_{0}}\right|}{M_{p}^{2}} \\
& =0 \\
V_{\text {False }} & =|F|^{2}-3 \frac{\left|W_{0}+\Lambda^{3} e^{\frac{2 \pi i}{n} k}\right|}{M_{p}^{2}} \\
& \neq 0 \quad \text { for } k \neq k_{0} \\
\epsilon & =V_{\text {False }}-V_{\text {True }} \\
& =-3 \frac{\left|W_{0}+\Lambda^{3} e^{\frac{2 \pi i}{n} k}\right|}{M_{p}^{2}}+3 \frac{\left|W_{0}+\Lambda^{3} e^{\frac{2 \pi i}{n} k_{0}}\right|}{M_{p}^{2}} \\
& \sim \frac{\Lambda^{3}}{M_{p}^{2}} .
\end{aligned}
$$

Here $\Lambda$ and $F$ denotes the scale of the dynamical sector and the supersymmetry breaking parameter. (Note that the above equation gives the essential part of the scalar potential for our discussion but is not the full description of the exact scalar potential.) Other vacua $\left(k \neq k_{0}\right)$ must have non-zero vacuum energy and it is estimated to be of order $\sim \sigma^{2} / M_{p}^{2}$. In fact, this satisfies the required condition(3.1) for the safe decay of the cosmological domain wall. In this case, the domain wall problem is solved without introducing an extra breaking term nor the fine-tuning of the parameter.

\section{Model for inflation}

The third example is the thermal inflation model of Asaka et al[3]. This model is proposed by T.Asaka, J.Hashiba, M.Kawasaki and T.Yanagida as a solution for the cosmological moduli problem in gauge-mediated supersymmetry breaking theories. In ref.[3], $Z_{n+3}$ symmetry is imposed on the superpotential for the flaton. The vacua are degenerated and has $Z_{n+3}$ phase thus an explicit breaking of the discrete symmetry is needed to remove the domain wall. In ref.[3] a small term $\alpha X$ was added by hand to the superpotential so that the discrete symmetry is explicitly broken. However, as we have seen

\footnotetext{
${ }^{2}$ To avoid the appearance of the negative energy state, supersymmetry breaking parameter $F$ should satisfy the condition $V=|F|^{2}-3\left|W_{t o t}\right|^{2} / M_{p}^{2} \geq 0$ for any $\mathrm{k}$-th vacuum. It is also required that the cancellation works for the lowest energy state of the $\mathrm{k}$-th vacuum.)
} 
above, such an unnatural term is not necessitated if $Z_{n}^{R}$ is assigned instead of $Z_{n+3}$. (See eq.(2.9).)

Next-to-minimal supersymmetric Standard Model

The last example is the next-to-minimal supersymmetric Standard Model (NMSSM). This model contains an additional singlet Higgs superfield and $Z_{3}^{R}$ symmetry which transforms every chiral superfield $\Phi$. The $\mu$-term is generated because the singlet that parametrizes the $\mu$-term gains non-zero vacuum expectation value. In this model, the natural scale for the domain wall is the weak scale. Although the energy gap is produced in the same way by the constant term in the superpotential, one should consider further constraint coming from primordial nucleosynthesis because the scale of the wall is smaller than $10^{5} \mathrm{GeV}$ (see ref.[2]). In fact the weak-scale walls decay after nucleosynthesis and the entropy produced when the wall collide is dumped into all the decay products. In this case, because of this additional constraint, the solution is not automatic. One possibility is to raise the surface energy density of the wall $\sigma$ above $10^{5} \mathrm{GeV}$. However, it requires fine-tuning of parameters because the $\mu$-term is already fixed at the weak scale. Another possibility is to introduce a non-renormalizable term that explicitly breaks the symmetry. On the whole, unlike other walls discussed above, the domain wall in NMSSM requires additional mechanisms or fine-tunings of the parameters to evade the cosmological domain wall problem.

The origin of the constant term

Although we did not mentioned about the origin of the constant term $W_{0}$ in the above discussions, it would be fair to say that such contribution is very common. One may think that the constant term appears from another dynamical sector which have the potential $\Lambda^{3} e^{\frac{2 \pi i}{n}}$. The most obvious example is the simple SYM with the product gauge group $S U\left(N_{1}\right) \times S U\left(N_{2}\right)$. The resulting superpotential is expected to be of the form: $W=\Lambda_{1}^{3} e^{\frac{2 \pi i}{N_{1}} k_{1}}+\Lambda_{2}^{3} e^{\frac{2 \pi i}{N_{2}} k_{2}}$, where $\Lambda_{1,2}$ is the dynamical scale of each gauge sector. Although we did not introduced an explicit constant term in this case, the vacuum degeneracy is obviously lifted when supergravity is turned on. 


\section{Conclusion and discussions}

In general it is quite difficult to find a successful model for supersymmetry breaking without introducing unnatural components. In this paper we have shown that the existence of the domain wall does not further constrain these models provided the surface energy density of the wall is not so low as the weak scale. Our point is that the cosmological constant should be cancelled by adding a constant term to the superpotential. This constant explicitly breaks any continuous or discrete R-symmetry, and lifts the degenerated vacua.

In this paper we have concerned with two types of walls that is related to two kinds of

$Z_{n}^{R}$ symmetry. One is the explicit symmetry of the Lagrangian, and the other appears as the consequence of the breakdown of the continuous $U(1)_{R}$ symmetry through anomaly. The wall of the latter type is especially important because it appears in almost all the dynamical supersymmetry breaking models.

\section{Acknowledgment}

We are very grateful to T.Asaka for many useful discussions.

\section{References}

[1] B. Chibisov and M. Shifman, hep-th/9706141; A. Kovner, M. Shifman and A. Smilga, Phys.Rev.D56(1997)7990; G. Dvali and M. Shifman, Phys.Lett.B396(1997)64

[2] S.A.Abel, S.Sarker and P.L.White, Nucl.Phys.B454(1995)663

[3] T.Asaka, J.Hashiba, M.Kawasaki and T.Yanagida, hep-ph/9711501

[4] J.Bagger, E.Poppitz and L.Randall, Nucl.Phys.B426(1994)3

[5] T.Matsuda, hep-ph/9710230

[6] A.Vilenkin, Phys.Rev.D23(1981)852 
[7] M.Dine and A.E.Nelson, Phys.Rev.D48(1993)1277; M.Dine, A.E.Nelson and Y.Shirman, Phys.Rev.D51(1995); M.Dine, A.E.Nelson,Y.Nir and Y.Shirman, Phys.Rev.D53(1996)2658 